\title{
Les conflits d'infrastructures en lle de France. Des révélateurs des imperfections de la décision publique dans les espaces ruraux et périurbains*
}

\section{Conflicts over Public Utility Infrastructure in lle-de-France: signal of imperfections in public decision-making process in French rural and periurban areas}

\author{
Hai-Vu PHAM** \\ Université Paris Dauphine et SADAPT, INRA \\ vxphvu@free.fr \\ Thierry KIRAT \\ CNRS, IRISSO et Université Paris Dauphine \\ thierry.kirat@dauphine.fr
}

\author{
André TORRE \\ SAD-APT, INRA et Agroparistech \\ torre@agroparistech.fr
}

Mots-clefs : calcul économique, conflits, Ile-de-France, infrastructures, utilité publique

Keywords : conflicts, economic valuation, Ile-de-France, infrastructures, public utility

Classification JEL : H54, K23, R53, D74

\footnotetext{
*Ce travail a été réalisé dans le cadre du projet ANR COVER, qui portait sur les dynamiques de conflictualité dans différentes zones de l'espace national.

*** Auteur correspondant.
} 


\section{Résumé}

La région Ile-de-France connaît une pression foncière importante due aux besoins d'espaces urbanisables et à la nécessité d'infrastructures à destination de la métropole parisienne. Or, les marges de manœuvre pour la poursuite de l'artificialisation des sols et à la création d'aménagements urbains tendent à se réduire. L'article analyse les conflits d'infrastructures qui traversent la région et éclairer leur lien au processus de décision publique. L'étude empirique menée sur le contentieux montre que les conflits d'infrastructures sont essentiellement localisés dans les espaces périurbains, dans des communes dont la population est relativement aisée. Ils sont l'expression d'une prise de parole de groupes de riverains désireux de faire entendre leur opposition à des projets qui ne leur paraissent pas acceptables en l'état, mais également de la lutte entre intérêts liés à l'urbanisation et volonté de préservation de l'espace. Ces conflits mettent en évidence la complexité et les ambiguïtés du processus de décision publique.

\section{Abstract}

The article offers an empirically-based analysis of conflicts over public utility infrastructures in the Paris region. It sheds some light on public decision-making process and on the role conflicts play in the process of land availability. Area tends to be lessened due to economic development and urban sprawling. The need of space for urbanization is undeniable, but the margin for operations is reducing because of growing scarcity of available land. Empirical evidence based on courts records show that conflicts on infrastructures occur mainly in peri-urban spaces, in relatively small and wealthy towns. Being proof of the local inhabitant's non satisfaction, the conflicts could be seen as an echo of the struggle between needs of urbanization and land preservation. The article also spots on the complexity and the ambiguity of the public decision-making process, by offering a critical lecture of its principles and its application in terms of infrastructure project.

\section{-1 - \\ Introduction}

Première agglomération française et deuxième agglomération européenne (GILLY, 2007), la région Ile-de-France connaît une pression foncière importante, due à la fois à l'accroissement de ses activités et à l'étalement urbain qui la caractérise. Les espaces agricoles ou naturels, qui constituent autant de réserves pour l'urbanisation, se voient progressivement remplacés par des habitations ou par des infrastructures de natures diverses, au service de la ville et indispensables à la vie et à la dynamique de la région, qu'il s'agisse des activités de transport (routes, autoroutes, voies ferrées et de tramways), de la production et de l'acheminement de l'énergie, du traitement des déchets, ou de constructions en lien avec les activités urbaines.

Avec une population de onze millions d'habitants (MATHIEU, 2005) et quelque vingt-trois millions de déplacements quotidiens (ORIZET, 2003), la croissance de la région nécessite la création permanente d'aménagements et d'infrastructures spécifiques, ainsi que la consommation d'espaces jusque-là dédiés à d'autres fonctions, telles que les activités de production, de loisirs, ou de nature. Pourtant, quatre-vingt pour cent de la superficie francilienne est encore occupée par des espaces ruraux, c'est-à-dire des forêts et des espaces agricoles (AGRESTE, 2011) et si l'on se réfère au seul coefficient d'occupation des sols, l'Ile-de-France demeure une région à dominante rurale, avec des zones écologiques et environnementales fortement protégées. Toutefois, les espaces agricoles franciliens connaissent une tendance forte à la contraction, 
sous l'effet d'une urbanisation périurbaine majoritairement radioconcentrique, mais qui tend à se diffuser de plus en plus loin de la petite couronne (CENTRE D'ANALYSE STRATÉGIQUE, 2012 ; IAURIF, 2001 ; SEGESA/DREIF/DRIAF, 2005).

Le besoin en espaces urbanisables dans les communes périurbaines franciliennes à solde migratoire positif est indéniable, tout comme la nécessité d'infrastructures à destination de la métropole parisienne. Toutefois, les marges de manœuvre relatives à la poursuite de l'artificialisation des sols, à la création d'aménagements liés à la mobilité domicile-travail et aux services urbains tendent à se réduire. La raison n'en est pas seulement la raréfaction des zones disponibles en périphérie de Paris et le prix croissant des ressources foncières non artificialisées, mais aussi l'intervention des résidents périurbains, qui souhaitent conserver leur cadre de vie et des paysages non ou faiblement urbanisés. Dans un tel contexte, souvent qualifié un peu rapidement de " Nimby « ${ }^{1}$, il est facile de comprendre pourquoi les projets de création d'infrastructures en Ile-de-France rencontrent fréquemment, comme dans d'autres régions confrontées au problème de l'étalement urbain, de vives oppositions. Il est intéressant, à ce titre, de noter l'importance des conflits liés à la mise en place d'infrastructures publiques, qui se jouent sur des zones limitrophes de l'espace rural ou d'origine agricole et que l'on peut classer en infrastructures linéaires (routes, autoroutes, lignes de transport d'électricité, voies ferrées) et non linéaires (centres de traitement des déchets, établissements pénitentiaires ou de jeunes délinquants, etc.). Ces conflits révèlent toute la difficulté de l'accroissement et de l'étalement des activités urbaines, face à des résidents décidés à préserver leur cadre de vie.

Bien qu'un nombre croissant de chercheurs et d'équipes s'y attache, la conflictualité des usages des espaces reste un domaine peu investigué dans les sciences sociales. Cet article s'inscrit dans le cadre des travaux qui, depuis quelques années, se développent autour de l'analyse des conflits d'usage des espaces, dans différents champs des sciences sociales: la géographie sociale (BOURDIN et al., 2006 ; CADORET, 2006 ; CHARLIER, 1999 ; DARLY, 2009 ; DARLY et TORRE, 2008 ; DOYON, 2007 ; LECOURT, 2003 ; MELE et al., 2003), la géographie urbaine (JOERIN et al., 2005 ; TRUDELLE 2003), la sociologie du droit (KIRAT et MELOT, 2006 ; MELOT, 2009), l'économie régionale (BOUbA-Olga et al., 2009 ; KirAT et TORRE, 2006 ; CARON et TORRE 2006 ; TORRE, 2010) ou l'économie publique (Jeanneaux, 2006 ; JEANnEaux et PERrier-Cornet, 2009 ; PHAM et TORRE, 2012); voire de manière transversale (TORRE et al., 2010). La conflictualité concernant les usages des sols présente deux caractéristiques majeures : elle révèle les rivalités et les oppositions, souvent organisées et récurrentes, dans les processus de mutation des espaces, et elle exerce un effet en retour sur les transformations et les vocations des territoires (TORRE et al., 2006) ; nous nous concentrons ici sur la première dimension.

L'article procède comme suit : la section 1 met en évidence les résultats empiriques obtenus à partir de l'étude des conflits sur les aménagements et infrastructures dans la région Ile-de-France, en détaillant leurs caractéristiques et les acteurs engagés, ainsi que leur évolution géographique et territoriale. La section 2 se concentre sur le caractère " préventif » des conflits qui prennent naissance avant la réalisation des infrastructures et mettent en jeu les procédures de décision publique. La section 3 


\section{présente un cas d'aménagement routier particulièrement conflictuel qui illustre les points développés dans la section précédente.}

\section{OBSERVER LES CONFLITS : SOURCES ET MÉTHODES}

L'analyse de la conflictualité nécessite de disposer de données sur les conflits proprement dits, afin de prendre la mesure empirique des phénomènes d'opposition, du mode d'expression des conflits, de leurs causes et de leurs origines ou des solutions qui y sont apportées. Or, les données concernant l'état de la conflictualité sont peu nombreuses et souvent lacunaires pour deux raisons principales : le faible intérêt pour ce domaine jusqu'aux années 2000, ainsi que la forme complexe prise par les conflits, lesquels revêtent des expressions diverses (tribunaux, médiatisation, manifestations...), proscrivent toute représentation simple et conduisent à faire appel à différentes disciplines dans leur définition. Le conflit analysé est un construit qui s'élabore souvent à partir d'informations recueillies auprès de différentes sources.

En France comme dans d'autres pays, aucun système statistique n'est dédié aux conflits d'usage des espaces et des ressources territorialisées (paysages, etc.). Un groupe de chercheurs de I'INRA, du CNRS et de I'Université, venant de disciplines différentes (économie, sociologie, géographie, psycho-sociologie), parmi lesquels les auteurs du présent article, soutenus par des financements publics, a mis en place une base de données dédiée aux conflits d'usage dans les territoires de France. Originale et répondant à un souci d'exhaustivité dans le recensement des conflits, elle est alimentée par trois types de sources : presse quotidienne régionale, contentieux et enquêtes qualitatives. Les données issues des deux premières sources sont rendues compatibles par une nomenclature et des variables communes, issues d'une élaboration collective ${ }^{2}$, auxquelles sont associées des données de contexte socio-économique. L'échelle territoriale retenue est celle de la commune.

Notre propos est ici limité aux données issues du contentieux, qui nous semblent les plus aptes à un premier traitement de la question des infrastructures, et révèlent comment les riverains s'adressent aux tribunaux, afin d'éviter l'installation d'infrastructures non conformes à leurs attentes en matière de cadre de vie. Les données sont issues d'un travail de recueil de textes (décisions de justice) et d'exploitation sous la forme d'une extraction des données pertinentes via un codage des décisions. La base documentaire LamylineReflex est la principale source utilisée, dans la mesure où elle est exhaustive sur les décisions du Conseil $d^{\prime}$ Etat et des Cours administratives d'appel pour le contentieux administratif, et sur celles de la Cour de cassation pour le contentieux civil et pénal. Elle permet une recherche des décisions pertinentes en mode texte, par combinaison de mots-clés et des opérateurs booléens (voir KIRAT et MELOT, 2004). Chaque décision constitue une observation pour la base. Les types de conflits sont distingués par leur objet: une usine polluante dont le fonctionnement est contesté ou encore un projet de déviation de route refusé par des riverains. Les décisions de justice permettent d'identifier les arguments avancés par les requérants, c'est-à-dire, en termes juridiques, les «moyens» auxquels la juridiction saisie est tenue de répondre.

La définition des confits d'usage et de voisinage repose sur trois éléments :

- la distinction entre conflits et tensions. Par rapport à la tension, le conflit traduit le passage d'un seuil qualitatif, qui correspond à l'engagement des parties dans la relation conflictuelle et a pour but de crédibiliser leurs positions. L'engagement implique un coût, monétaire ou hédonique, et peut prendre différentes formes : le recours en justice, la publicisation (différend porté devant des instances publiques ou des services de l'Etat), la médiatisation (différend porté devant les média), les voies de faits ou la confrontation verbale, la destruction de biens ou d'infrastructures, la production de signes (interdiction d'accès, barrières...).

- I'inscription dans le territoire. Les conflits d'usage de l'espace reposent sur une base physique, se déroulent entre voisins et prennent naissance autour de biens supports matériels ou immatériels localisés. Ils s'inscrivent dans un cadre institutionnel déterminé à la fois par les jeux et les règles des instances locales et supra-locales. 
- la matérialité. Les conflits auxquels nous nous intéressons sont liés à une matérialité d'actes réalisés ou anticipés. Les oppositions de personnes ou de groupes se réfèrent à des objets concrets, à des actes techniques en cours ou à venir et se traduisent par des actions concrètes.

Les projets d'aménagements et d'infrastructures ont été identifiés comme l'objet matériel déclencheur du conflit : installation d'une antenne relais pour la téléphonie mobile, construction d'une route, etc. Cet objet matériel peut être formulé en termes juridiques dans un registre différent, par exemple lorsque les requérants contestent une décision de modification du plan local d'urbanisme dont la finalité est à terme de rendre possible l'implantation d'une infrastructure. La base documentaire permet de saisir, pour chaque conflit, son objet matériel et le domaine juridique de la requête.

\section{- 2 - \\ La réalité du contentieux administratif des conflits d'infrastructures en Ile-de-France Titre de la première partie}

Cette section est consacrée à une présentation des conflits franciliens qui trouvent une expression devant la justice administrative en matière d'aménagements et d'infrastructures durant la période 1981-2005. Les données utilisées sont issues du contentieux administratif localisé en Ile-de-France du 1er janvier 1981 au 31 décembre 2005. Elles consistent en décisions rendues par le Conseil d'Etat et, à partir de leur création en 1989, par les Cours administratives d'appel de Paris et de Versailles, sur des conflits localisés dans les six départements de l'Ile-de-France, Paris étant exclu du champ de l'étude au regard de la spécificité de la ville-capitale.

Ces conflits, principalement situés dans les espaces périurbains, révèlent les oppositions entre les différents usages du sol, ainsi que l'action menée par des groupes de résidents qui désirent préserver leur zone d'habitat de l'urbanisation et de la construction d'infrastructures liées aux fonctions de la ville. Exprimés sous une forme particulière, qui est le langage du droit, les conflits d'infrastructure ici présentés témoignent des préoccupations locales quant aux localisations futures des équipements publics. Ils révèlent que le souci de la détérioration du bien-être des riverains est à l'origine des conflits provoqués par le projet d'installation d'une infrastructure indésirable à proximité. Souvent considérées comme Nimby, ces oppositions ne manquent pourtant pas de fondements objectifs, et les argumentaires sont soignés, même si l'on ne peut exclure les conduites opportunistes bien sûr.

\subsection{Quelques informations sur l'évolution géographique des conflits d'usage de l'espace en Ile-de-France}

L'évolution des conflits dans l'espace est un bon indicateur du processus de périurbanisation de la région Ile-de-France, processus qui se heurte à de nombreuses oppositions des populations déjà installées. Nos travaux, menés à la fois sur le contentieux et l'analyse de la Presse Quotidienne Régionale (PQR, diverses éditions 
du journal Le Parisien) et publiés par ailleurs (DARLY et TORRE ; 2008 ; DARLY, 2009 ; TORRE et al., 2010), montrent que les conflits d'infrastructures ne sont pas distribués au hasard dans l'espace francilien. Au contraire, ils se concentrent dans la zone frontalière du grand Paris urbain : la partie fortement urbanisée de l'agglomération parisienne (Paris et ses trois départements limitrophes, soit la "petite couronne») semble être faiblement touchée. Les conflits témoignent de la contrainte spatiale à laquelle est confrontée l'agglomération parisienne pour s'agrandir et mettre en place des infrastructures nécessaires à l'aménagement du projet urbain.

Au cours des trente dernières années, les conflits se multiplient dans différents lieux de la région, tous situés à la frontière de la petite couronne. Il s'agit de communes périurbaines à l'interface entre l'agglomération parisienne et les espaces naturels et agricoles. Ces communes ont un rythme d'urbanisation assez élevé (en moyenne plus de cinquante autorisations de construction délivrées par an) et sont appelées à devenir urbaines. Les conflits montrent que l'extension de la ville ne se fait pas toujours facilement, car les tentatives d'aménager des infrastructures se confrontent à des oppositions organisées des riverains qui désirent préserver l'environnement ou le cadre de vie.

Les infrastructures mises en cause sont diverses, mais leur géographie reflète nettement les périodes d'aménagement de l'Ile-de-France, que l'on peut caractériser de manière schématique. Ainsi, la période 1985-1995 est marquée par la construction de l'autoroute A86 et les autoroutes et routes qui lui sont liées, sur des tracés controversés, tandis que la période 1995-2005 révèle l'opposition forte des riverains aux projets d'élargissement de deux aéroports Roissy et Orly. Aujourd'hui le bouclage de l'A86 est achevé, mais ses travaux d'aménagement ont commencé en 1968. Il a donc fallu quarante ans pour finaliser une voie d'une soixantaine de kilomètres autour du grand Paris.

Le croisement des résultats issus du contentieux et de données socio-économiques révèle que les conflits d'infrastructures émergent le plus souvent dans les communes relativement aisées, mais où le taux d'urbanisation est important. Une population communale d'environ trente-cinq mille habitants dont 30\% sont des cadres ou exercent une profession intermédiaire ; seulement $15 \%$ d'ouvriers et d'employés ; un nombre effectif de cent quarante-cinq permis de construire délivrés chaque année ; un revenu individuel moyen annuel de $33.000 €$; une distance d'une trentaine de $\mathrm{km}$ de Paris : tels sont les traits d'une commune francilienne type - lieu des conflits d'infrastructures.

La principale concentration de conflits issus de l'analyse de la PQR se trouve dans l'Ouest Parisien, qui concentre les populations les plus aisées et des communes fortement urbanisées. La mise en parallèle des deux sources (PQR et contentieux administratif) révèle les stratégies différentes des acteurs en matière de déclenchement des relations conflictuelles. Dans les territoires sous pression directe de l'agglomération parisienne, à la frontière urbaine, les stratégies d'affrontement (guerre juridique) se révèlent dominantes, alors que dans les zones un peu plus lointaines, c'est plutôt la publicisation par la manifestation publique ou la couverture médiatique qui fait 
office de sonnette d'alarme à la détection des projets d'aménagement. Si les projets les plus controversés se localisent sur le périurbain assez peuplé, à la frontière urbaine et en cours d'urbanisation, il semble y avoir une gradation dans l'engagement dans le conflit, qui part de la mobilisation des médias à l'annonce des projets et se poursuit par le recours à la justice à partir de la mise en place de la procédure de déclaration d'utilité publique.

\subsection{Intérêt d'une analyse des conflits d'infrastructures}

Quand ils sont analysés par le prisme du contentieux, les conflits localisés en Ilede-France relèvent principalement du droit administratif et se concentrent sur trois domaines : l'urbanisme, les installations classées soumises à un régime d'autorisation et de contrôle par l'administration et les aménagements et infrastructures d'utilité publique (PHAM et KIRAT, 2008). Les contestations visent les documents ou la mise en place et le fonctionnement des équipements publics et privés, pendant que la discussion de fond, derrière la mise en forme juridique de ces conflits, porte souvent sur un désaccord dans l'allocation des droits d'usage de l'espace. Ainsi la transformation d'un sol non-constructible en sol urbanisable, la validation d'un projet d'infrastructure près d'une zone naturelle, la création d'une usine industrielle limitrophe des résidences pourraient alimenter des conflits qui vont jusqu'au Conseil d'Etat, car elles laissent la place à un débat sur les « risques de nuisance « tout d'abord et le devenir du territoire ensuite.

Les conflits d'infrastructures nécessitent une attention particulière, car ils concernent souvent des projets à fort impact sur la population et sur l'environnement et se révèlent fortement consommateurs de sols. L'analyse du contentieux montre en effet que les infrastructures contestées touchent souvent plusieurs communes, généralement périurbaines, plutôt aisées et avec un rythme d'urbanisation assez élevé. Les équipements publics qui font l'objet de la contestation sont construits ou projetés au bénéfice des habitants de ces communes, mais également de ceux des pôles urbains auxquels elles appartiennent, en particulier Paris. Les conflits d'infrastructures s'inscrivent alors dans des relations territoriales à différentes échelles. Le projet peut rester local mais son utilité porte, dans de nombreux cas, sur l'échelle régionale, voire nationale.

De plus, à la différence des deux autres domaines forts de la conflictualité en Ile-de-France (urbanisme et industrie), les projets d'infrastructure sont liés à la question de l'utilité publique, qui se situe à l'interface du droit administratif et du calcul économique public et ne manque pas de soulever le problème de l'équité dans l'exposition aux nuisances (KIRAT et LEVRATTO, 2008). La méthode coûts-bénéfices socio-économiques appliquée en la matière est un sujet controversé pour le calcul économique lui-même (entre autres GibLIN 2004) et le déroulement du processus de prise de décision publique. L'étude de cas présentée plus loin en donnera des éléments empiriques. 


\subsection{Description des acteurs et des objets de conflits d'infrastructure}

Les principaux objets des conflits d'infrastructure sur la période 1981-2005 sont présentés dans le Tableau 1

\section{Tableau 1 - Principaux objets des conflits d'infrastructure en Ile-de-France, 1981-2005}

\begin{tabular}{|l|c|}
\hline Objets des conflits & $\begin{array}{l}\text { Nombre de décisions des juges } \\
\text { administratifs }\end{array}$ \\
\hline Infrastructure routière & 36 \\
\hline Etablissement public & 17 \\
\hline $\begin{array}{l}\text { Infrastructure de transport \& production } \\
\text { d'énergie }\end{array}$ & 10 \\
\hline Infrastructure aéroportuaire & 8 \\
\hline Infrastructure ferroviaire & 4 \\
\hline Infrastructure fluviale & 1 \\
\hline Infrastructure de télécommunication & 1 \\
\hline Divers & 1 \\
\hline Total & 78 \\
\hline
\end{tabular}

Basés sur l'iinventaire des décisions de justice extraites de la base LamylineReflex, le tableau recense les réponses des juges administratifs aux requêtes qui leur sont adressées par les protagonistes et les opposants des projets d'infrastructure contestés. On trouve ainsi, au premier rang de la conflictualité, les infrastructures routières, dont les opposants mettent en avant les conséquences sur la pollution de l'air, le bruit, la poussière, l'impact paysager, ainsi que la dévalorisation immobilière. Plus concrètement, il s'agit avant tout des oppositions au bouclage de l'autoroute A86 (deuxième périphérique de Paris) et aux aménagements des routes et autoroutes correspondantes.

Au deuxième rang apparait la création d'établissements publics, qui concerne aussi bien des établissements aux impacts positifs que communément reconnus comme négatifs. On trouve ainsi, à côté des stations d'épuration - exutoires des eaux usées les projets de construction d'hôtels de ville, de casernes militaires et de gendarmeries ou de salles polyvalentes communales. La contestation de ces établissements « non polluants » est principalement menée par des associations dans le souci de protéger des espaces boisés ou naturels que menace le projet d'aménagement. La dimension d'anticipation de ces conflits est patente : ce qui est jugé discutable, ce n'est pas l'infrastructure elle-même, mais le processus d'urbanisation qu'elle pourrait entrainer.

Le cas des infrastructures de production et de transport de l'énergie est un peu différent. C'est la dangerosité de ces équipements (ligne de haute tension, réseau de gaz) qui est mise en avant, ainsi que la servitude d'utilité publique qui y est liée et nécessite un espace dédié. Les opposants à ce type d'infrastructure sont avant 
tout des associations de riverains, composées à la fois des propriétaires expropriés et d'habitants voisins de l'espace du projet. Les infrastructures aéroportuaires sont le dernier objet emblématique de la région. En effet, les bruits de décollage et d'atterrissage des avions pendant la nuit font l'objet de conflits durables entre les riverains, les autorités aéroportuaires et les compagnies aériennes présentes à Roissy et Orly depuis des décennies. Nous ne prenons pas en compte ici ce type de conflits liés au service de transport aérien, mais ils impactent directement les infrastructures de transport aérien et leur construction. Dans une telle situation, la création de nouvelles infrastructures aéroportuaires (extension des pistes, construction et/ou modification des bâtiments) représente la goutte d'eau qui fait déborder le vase et provoque les conflits les plus explosifs de la région en termes de mobilisation d'acteurs, de médiatisation et de durée (cf. FABUREL 2003).

La mesure des conflits par le seul nombre de décisions de justices est partielle, car le juge peut répondre à plusieurs requêtes ou trancher un conflit relatif à plusieurs parties dans un seul jugement. C'est pourquoi nous proposons de mesurer l'intensité des conflits par le nombre de parties engagées dans un conflit et le nombre de requêtes traitées dans chaque juridiction ( $c f$. Graphique 1). Les conflits les plus intenses c'est-à-dire ceux qui donnent lieu au plus grand nombre de recours juridiques et qui concernent le plus de parties - se trouvent en haut à droite du Graphique. Ils sont liés à l'aménagement des aéroports, des lignes ferroviaires et routières. La construction des lignes de TGV, par exemple, mobilise plus de cinq parties et fait en moyenne l'objet de plus de deux requêtes devant le juge administratif. Un recours juridique à l'encontre des projets aéroportuaires engage environ six parties et donne lieu en moyenne à deux requêtes.

Comme le montre le tableau 2, les parties engagées dans les conflits d'infrastructures se répartissent, en Ile-de-France, en deux catégories : les acteurs locaux d'une part (associations, collectivités territoriales et particuliers) et les pouvoirs publics (collectivités territoriales et leurs établissements publics, préfets, ministres) d'autre part. Si les premiers sont principalement demandeurs de décisions de justice (sur 208 demandes, 174 proviennent de ces acteurs, soit un taux de $84 \%$ ), les seconds sont les plus contestés (137/208 soit $66 \%$ des cas contestés). Ce résultat n'est pas étonnant dans la mesure où les requêtes juridiques liées à l'aménagement des infrastructures consistent généralement à demander soit l'annulation d'un arrêté préfectoral de déclaration d'utilité publique, soit son rétablissement si le tribunal administratif a procédé à son annulation en première instance. La confrontation entre l'intérêt local et l'intérêt général est ainsi ici indéniable. En effet, trois quarts des conflits (157 contestations sur 208) portés devant les Cours administratives d'appel et le Conseil d'État mettent en jeu des confrontations entre acteurs locaux et pouvoirs publics. 


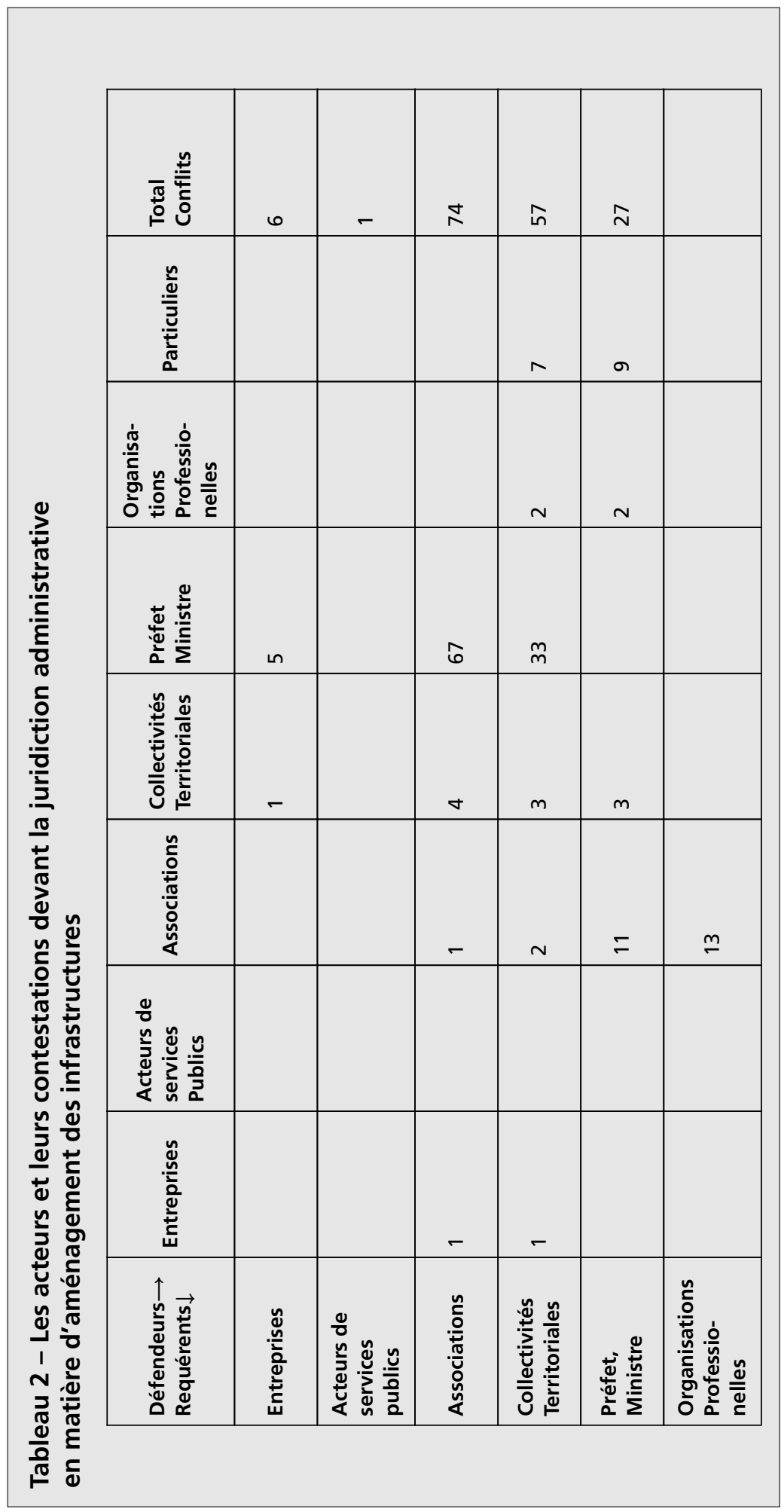


Hai-Vu PHAM, Thierry KIRAT, André TORRE

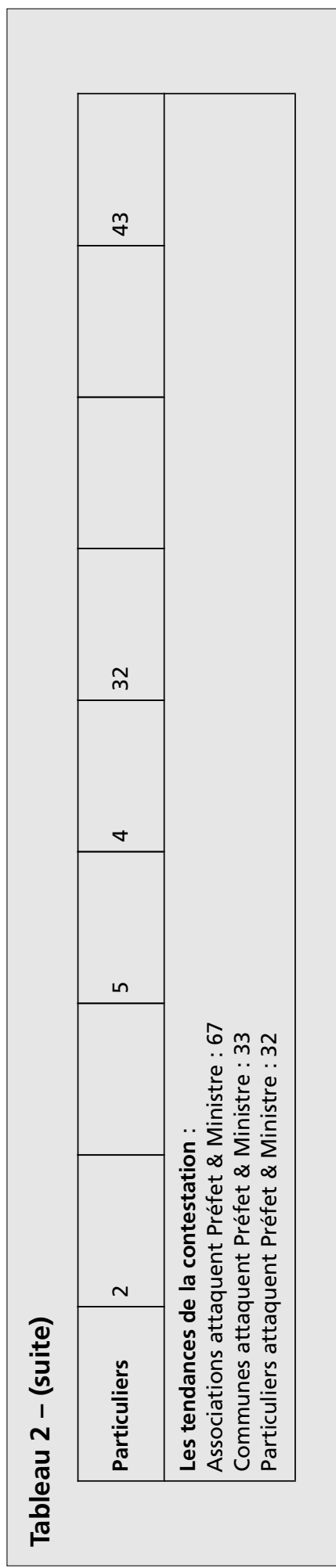




\section{Figure 1 - Positionnement des conflits d'infrastructures en lle-de-France selon leur intensité}

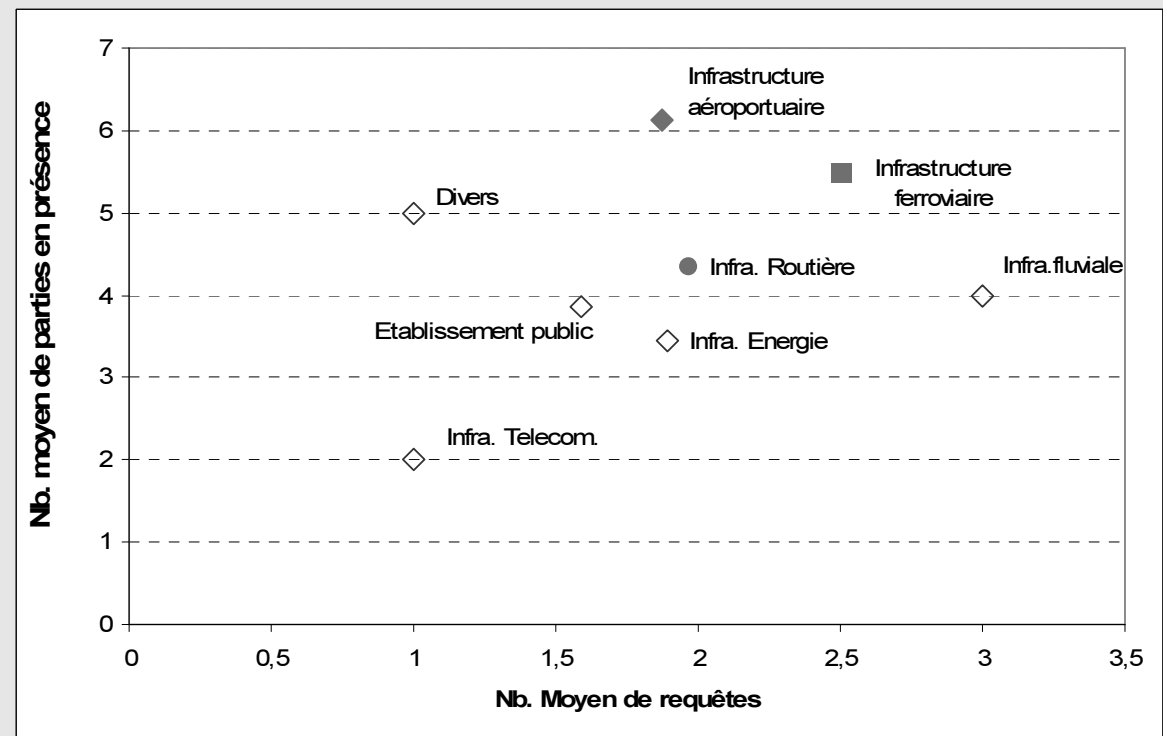

\section{- 3 - \\ Des conflits anticipés qui pèsent sur l'action publique}

Un examen attentif des cas présentés ci-dessus permet de qualifier un conflit d'infrastructure type, porté devant la juridiction administrative. En général, le conflit émerge de l'acte administratif qu'est la déclaration d'utilité publique d'un projet d'infrastructure, le plus souvent un équipement public, portée par l'administration au nom de l'intérêt général. Evidemment, le conflit n'émerge que lorsque les acteurs considèrent que la déclaration d'utilité publique ne prend pas en compte les tensions ou critiques exprimées lors des phases préalables de concertation. Des acteurs locaux s'opposent alors au projet et attaquent la déclaration d'utilité publique au nom des nuisances avérées, supposées ou anticipées de ce projet au niveau local.

Dans le domaine des infrastructures, comme d'ailleurs dans celui des activités industrielles ou extractives soumises à un régime de déclaration ou d'autorisation administrative, les conflits portés au contentieux en Ile-de-France sont essentiellement l'expression d'actions de contestation que l'on peut qualifier d'ex ante, c'est-à-dire dans lesquelles l'opposition porte sur le projet et non sur le bien ou l'équipement déjà mis en fonctionnement, et vise à empêcher sa construction effective. Ces oppositions manifestes peuvent se définir comme des " conflits d'anticipation ", qui visent à bloquer les projets contestés ou, du moins, à faire évoluer leur contenu dans un sens moins dommageable pour un certain nombre de parties prenantes. 
Cette caractéristique des conflits d'infrastructures vient du fait que les riverains se mobilisent très tôt par rapport aux projets de nouveaux aménagements et que leur capacité d'intervention se concentre sur les phases amont de l'opération. L'action en contentieux est permise par le principe de décision publique, à l'origine du projet, qui repose sur la déclaration d'utilité publique annonçant la mise en œuvre d'un projet d'infrastructure. L'enquête publique qui la précède ne discute pas des éventuels dommages à l'égard de la population locale et les suppose réparables ou compensables. Mais elle ne concerne qu'une partie de la population et ne peut révéler toutes les oppositions, qui s'expriment ensuite dans des conflits où les opposants défendent des intérêts non reconnus par la procédure de concertation.

\subsection{Enjeux analytiques}

La dynamique d'occupation des sols et de transformation des usages constitue une source importante de conflits d'usage, comme il est avéré dans la littérature, mais n'en est pas la condition suffisante. Les conflits d'usage sont également déterminés par les paramètres socioéconomiques caractéristiques de l'espace des projets de transformation des usages des sols, qu'il s'agisse d'espaces localisés ou d'espaces linéaires (cf. O'LEAR et al., 2005, WESTER-HERBER 2004).

Certains spécialistes de l'espace urbain ont adopté ce point de vue (JOERIN et al., 2005 ; TRUDELLE, 2003). Ils considèrent les conflits comme une relation antagonique entre deux ou plusieurs unités d'action, relation fortement liée à l'évolution ou à la perturbation des valeurs sociales. Mais ils prennent également en compte ce que nous qualifions de dimension " matérielle " du conflit et qui suppose son intégration dans un cadre spatialisé. Tensions et différends n'apparaissent pas par hasard sur le territoire: ils sont dépendants des caractéristiques des territoires occupés, en particulier des caractéristiques géophysiques (plaine, montagne, littoral, type de cultures ou d'occupations des sols. On n'oubliera pas non plus les dimensions sociodémographiques (structure par âge ou par catégories socioprofessionnelles de la population, solde démographique). Le travail empirique se doit de chercher à comprendre et à généraliser les caractéristiques de la conflictualité, mais aussi le rapport qu'elle entretient avec son espace.

L'étude du conflit peut être aussi menée dans le cadre plus large de l'analyse du processus de décision publique. Alors que les décisions publiques en matière d'aménagement du territoire sont fréquemment contestées en France, elle révèle à quel point ces procédures sont longues et peu efficaces en termes de supposée résolution des conflits. Certains aménageurs connaissent cette faiblesse depuis longtemps ; mais considèrent que le problème vient de la façon dont le projet est publiquement présenté. "La question qui se révèle est finalement moins de l'ordre méthodologique que de la capacité à convaincre de l'utilité du projet " (MERIAUX et al. 2002). Pourtant, la complexification de la procédure décisionnelle ne conduit pas à davantage d'acceptation locale, et l'on peut constater que les contestations visant directement la procédure sont nombreuses. En effet, si les riverains sont affectés par la décision publique, chercher à les convaincre sera chose impossible. La question sera plutôt de révéler les préjudices et d'ajuster la décision devant ces nouvelles 
variables. L'étude des conflits ouvre alors une piste de réflexion sur ces possibilités. Les travaux de TORRE et al. (2006) et BOUBA-OlgA et al. (2009) sur la capacité des populations d'un territoire à prendre la parole face une décision d'aménagement, ainsi que de JEANNEAUX (2006) sur le pouvoir d'ajustement de la décision publique des conflits d'usage, vont dans ce sens.

\subsection{Le processus de fabrication de la décision publique}

La procédure supervisée par l'administration vise à garantir l'utilité publique d'un projet, mais pas obligatoirement le fait qu'il s'agisse de la solution la plus satisfaisante pour la population locale, d'où le sentiment des opposants d'être souvent victimes d'une "mauvaise " décision (Raux et al., 2007). Pris dans un jeu dont les règles sont regardées a priori comme optimales - le processus décisionnel est considéré comme ouvert et contradictoire - les riverains manifestent leur désaccord au projet par le biais des conflits. De manière paradoxale, la réalité semble montrer que plus la procédure de concertation est minutieusement mise en application, plus le projet est contesté et facilement contestable.

Le choix d'un projet et la prise de décision en matière d'aménagement d'infrastructure par l'administration font l'objet d'un processus long et complexe, décrit dans l'encadré 1 . Ils sont fondés sur une appréciation à la fois quantitative et qualitative des avantages et désavantages du projet :

- les fondements de la décision apparaissent toujours comme un élément quantifiable on fait référence à un bilan positif des coûts et des avantages du projet projeté, réalisé par le calcul économique, afin d'en faire ressortir la légitimité au regard de l'utilité publique ;

- la validation des éléments qualitatifs est plus subtile. Elle passe par une procédure de concertation publique, durant laquelle on s'interroge sur les impacts du projet sur l'environnement.

Le processus comprend donc deux étapes, qui participent à l'appréciation de l'utilité publique: tout d'abord l'évaluation quantitative, réalisée en amont de la consultation du public, qui détermine par le calcul économique la rentabilité socioéconomique du projet et de ses variantes; puis l'enquête publique. Par ailleurs, lorsque l'importance du projet le justifie, un débat public peut être organisé. Dans cette deuxième séquence, il s'agit de faire un bilan qualitatif du projet à partir des observations recueillies auprès du public. Si les limites du calcul économique ont fait l'objet de nombreux commentaires de la part des économistes et aménageurs (voir par exemple Boiteux, 2001 ; GUESNERIE, 2006 ; MAURICE et Crozet, 2007), la discussion sur les problèmes posés par la procédure de concertation publique commence à prendre de l'ampleur, en particulier dans le cadre des recherches menées sur la place du débat public et de la démocratie participative dans l'aménagement du territoire (BLATRIX, 2009, 1997 ; BLATRIX et al. 2007). 


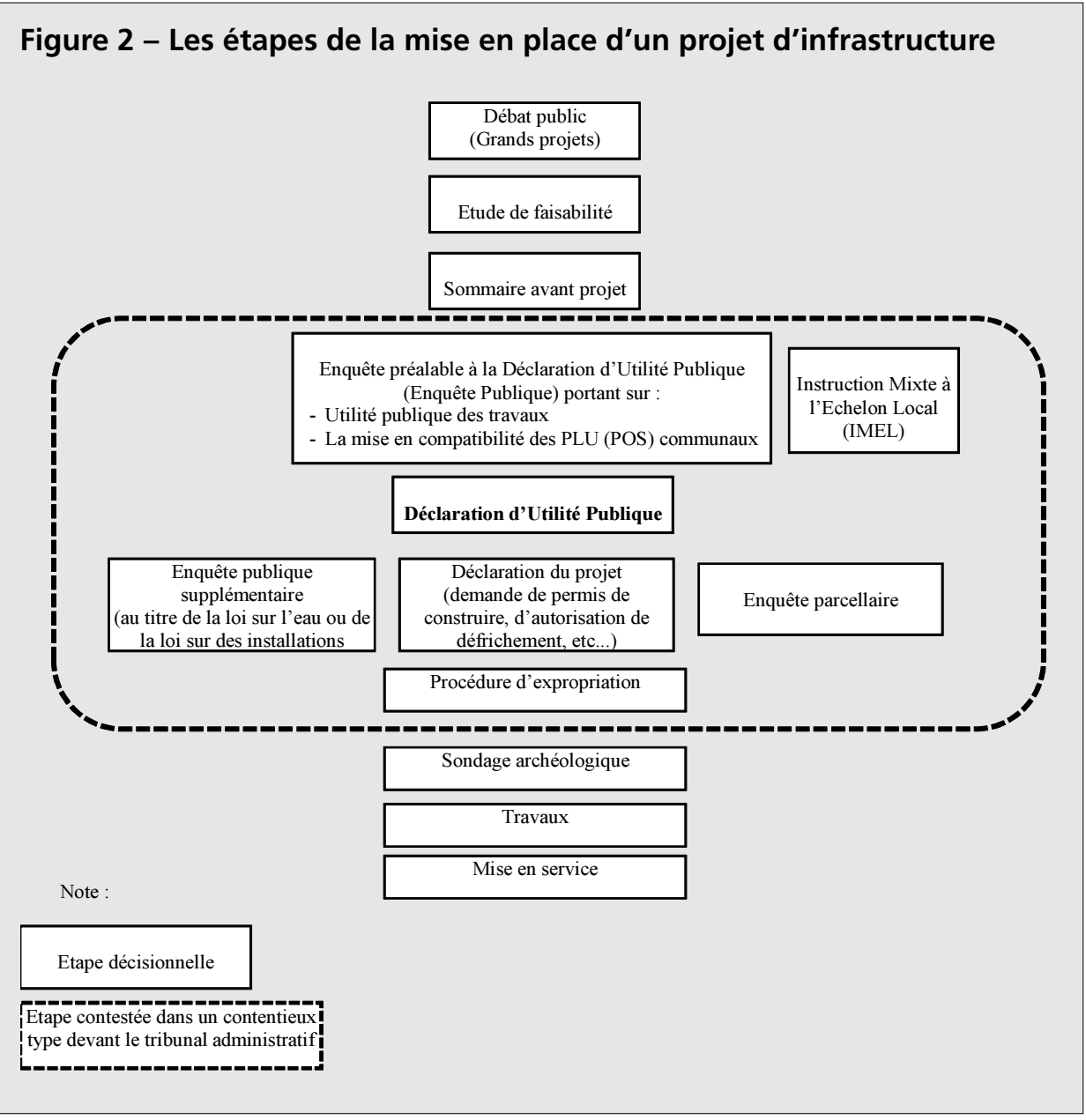

L'enquête publique préalable à la déclaration d'utilité publique a pour vocation de valider auprès de la population locale des éléments qualitatifs du projet, que le calcul économique public ne peut traiter. Ouverte par arrêté préfectoral et portée à la connaissance du public grâce à des affichages en préfecture et en mairies, elle constitue la source la plus importante de l'avis ex-ante des riverains sur le projet. Pendant l'enquête, les documents du projet sont également disposés en préfecture et en mairies à la consultation du public et les citoyens peuvent exprimer leurs opinions soit en assistant aux réunions d'enquête, soit par l'envoi des courriers auprès du responsable de l'enquête - le commissaire enquêteur. Ce dernier est chargé de la production de la seule ressource sur laquelle se fonde la décision administrative : le bilan des avis publics. Or, le commissaire enquêteur est souvent perçu comme un représentant de l'administration, une sorte de " délégué d'un projet » à l'écoute des riverains. Si, formellement, les calendriers de la présence du commissaire enquêteur et de l'ouverture des registres de consultation sont bien structurés, cette image peut expliquer l'atonie fréquente de l'expression du public. 


\section{L'ORGANISATION DES PROCÉDURES D'ENQUÊTE PUBLIQUE}

Pour empêcher que le soupçon ne porte sur la qualité de ses décisions techniques, le législateur n'a cessé, depuis trente ans, de multiplier les procédures qui permettent au public de s'exprimer. Une règle implicite semble présider à ces innovations successives : chaque procédure nouvelle va plus en amont que la précédente, descend plus avant dans les considérations techniques - et prend plus de temps.

Les premières enquêtes publiques avaient pour but de protéger les propriétaires contre les empiètements abusifs de l'administration. Le projet technique était déjà là, ficelé, indiscutable ; la discussion se faisait à la marge. La loi №83-630 du 12 juillet 1983 relative à la démocratisation des enquêtes publiques et de la protection de l'environnement (dite loi BOUCHARDEAU), va déjà plus loin, puisque le commissaire enquêteur enregistre les avis, qui peuvent déborder bien vite le cadre étroit de la solution technique proposée.

C'est ce débordement qui pousse en 1992 à la mise en place d'une nouvelle procédure, dite circulaire Bianco, propre aux autoroutes et aux voies ferroviaires. Ce texte ouvre la discussion bien avant la phase du tracé - soumis plus tard à l'enquête publique.

La loi du 27 février 2002 relative à la démocratie de proximité (codifiée par les articles L121-1 à L121-13 du code de I'environnement) institue une commission nationale du débat public en tant qu'entité administrative indépendante, chargée « de veiller au respect de la participation du public au processus d'élaboration des projets d'aménagement ou d'équipement d'intérêt national de l'Etat, des collectivités territoriales, des établissements publics et des personnes privées, relevant de catégories d'opérations dont la liste est fixée par décret en Conseil d'Etat, dès lors qu'ils présentent des forts enjeux socio-économiques ou ont des impacts significatifs sur l'environnement ou l'aménagement du territoire ».

Si I'on tient compte de toutes les étapes, la durée d'un projet peut atteindre dix ans voire plus.

De plus, le commissaire enquêteur ne vérifie pas si la procédure de concertation a rempli la fonction d'outil d'aide à la décision publique, mais seulement si elle a été correctement mise en place et si l'on peut tirer une conclusion " raisonnable » des éléments obtenus auprès du public. Sa mission n'est pas de faire un état des possibilités d'améliorer la décision, mais d'établir un bilan qualitatif des avis reçus au regard de l'intérêt et de l'impact du projet. Face à l'ensemble de ces éléments, il ne peut donner qu'une appréciation relative : des risques de nuisance ou de dommage pour les habitants locaux peuvent ainsi être traduits dans son avis comme des remarques peu contraignantes pour le décideur public. Par ailleurs, le Préfet est libre d'interpréter l'importance relative des avis positifs ou des remarques critiques ou réserves. De nombreux spécialistes estiment que l'enquête publique "demeure une procédure d'information et de consultation, sans pouvoir décisionnel conféré au public" (Hostiou et Helin, 1993). En tout état de cause, même si le commissaire enquêteur rend un avis défavorable, ce dernier n'a pas pour effet de remettre en cause la réalisation du projet : il contraint cependant à déplacer la déclaration d'utilité publique, du préfet ou du ministre vers le Conseil d'État ${ }^{3}$.

\subsection{Les conflits au regard de la décision publique}

Les conflits étudiés ici révèlent que les oppositions aux projets de construction ou d'aménagement d'infrastructures découlent à la fois des caractéristiques de la procédure mise en place et de l'évaluation quantitative réalisée par le biais du calcul économique. Les divers acteurs concernés par un projet cherchent, en entrant dans un 
processus conflictuel, à faire annuler ou à infléchir un projet qui leur est défavorable en s'appuyant sur deux registres :

- d'une part, les riverains attaquent le non respect des étapes procédurales de la décision. Le non affichage des panneaux d'enquête publique, l'inaccessibilité du dossier d'étude d'impact ou son manque de clarté, la non-disponibilité du commissaire enquêteur, ou encore l'absence des documents permettant une information complète du public sont des éléments très fréquents dans l'argument des auteurs de recours devant le juge administratif;

- d'autre part, ils s'interrogent sur des éléments non valorisables ignorés par le calcul économique du décideur public, notamment sur les principes d'équité ainsi que sur les impacts sur l'environnement ou sur le cadre de vie des résidents. Le développement du droit de l'environnement favorise cette stratégie, puisque les riverains se saisissent de ses éléments pour s'opposer à certains projets d'infrastructures.

Dans l'ensemble, toutefois, que soient mobilisés le registre de l'environnement ou la contestation des procédures de décision, c'est bien du cadre de vie dont il est question : les riverains cherchent à défendre leur environnement immédiat qui leur semblent menacé par le projet, en s'appuyant sur les ressources offertes par le droit, quand il ne s'agit pas de manifestations ou de mobilisation des médias.

Pour comprendre la place prise par les conflits dans le processus de développement territorial et le rôle qu'ils occupent dans l'expression des populations, on peut utiliser l'analyse de HiRSCHMAN (1970), qui offre un cadre de compréhension des modes de conflictualité, en particulier dans le cas des conflits d'infrastructures. Quand se produit un événement qui laisse insatisfait un agent (ou un groupe d'acteurs) $\left.{ }^{4}\right)^{5}$, on peut retenir trois solutions :

1) les acteurs qui considèrent que l'événement est non conforme à leurs intérêts peuvent décider de la solution d'exit, qui consiste soit à quitter le territoire et à se localiser dans une zone jugée plus favorable (exit spatial, ou vote avec les pieds), soit à quitter son activité économique. Il va sans dire que cette solution n'est pas toujours praticable, pour des raisons financières ou de prix du foncier (par exemple, il est difficile de revendre à un bon prix des terrains menacés par l'arrivée d'une infrastructure de transport) ;

2) une autre solution, la loyalty, peut être interprétée comme l'acceptation des décisions en cours, par une participation aux dynamiques et aux projets mis en place, ainsi que par l'absence d'opposition publique. Cette solution implique une fidélité à l'action publique, peu compatible avec les attaques contre le processus de décision publique ;

3) la troisième solution est celle du voice. Il s'agit de faire entendre sa voix et de s'opposer ainsi aux projets, aux décisions ou aux actions en cours : c'est la voie du conflit. On peut effectuer une distinction entre le voice individuel, plutôt réservé aux " petits» conflits, et le voice collectif, qui marque un élargissement de l'espace de 
concernement, ainsi que la mobilisation contre des projets de taille importante ou qui impliquent une reconfiguration forte des modalités de gouvernance au niveau local (DOWDING et al., 2000). Dans ce dernier cas, le processus de voice est souvent dirigé vers les pouvoirs publics (YOUNG, 1976), avec l'idée de remettre en question les décisions prises par les autorités et d'infléchir le processus de gouvernance. Le but est alors, soit de faire renoncer à de projets prévus ou déjà en cours, soit d'infléchir la décision et d'y incorporer une partie des arguments et des attentes des opposants, soit de modifier la composition des parties prenantes de la décision et d'y intégrer de nouveaux acteurs, justement ceux qui s'opposent au moyen du conflit. L'impact peut être considérable, l'ampleur du conflit et la mobilisation des personnes constituant des moyens importants d'infléchissement de la décision et de prise en compte de points de vue supplémentaires.

Il n'est pas impossible, mais nous ne disposons pas de la possibilité de confirmer empiriquement cette hypothèse, que les actions devant la justice administrative s'inscrivent dans une stratégie de recherche de positions fortes de négociation avec les autorités publiques et les maîtres d'ouvrage: la menace crédible de harcèlement procédurier peut en effet conduire les porteurs du projet à consentir des concessions plus fortes et rapides qu'en contexte de déroulement paisible du projet. Or, comme montré ci-dessus, les procédures de décision publique ne semblent pas être en mesure de fournir un cadre crédible de discussion controversée et ouverte des projets et de leur contenu technique.

\section{-4 - \\ Etude du cas du projet de déviation de la route D837 à Maisse}

Nous présentons ci-dessous un cas de conflit type, qui ressort de notre analyse du contentieux portant sur les décisions publiques concernant les infrastructures. Il s'agit de l'opposition des riverains à l'encontre du projet de déviation de la route D837 à Maisse, dans le département de l'Essonne. Il semble bien que les routes soient les infrastructures les plus controversées en Ile de France. Ce conflit particulier illustre de façon exemplaire la disproportion d'intérêts qui affecte les parties prenantes. En effet, tous les acteurs n'ont pas bénéficié ou supporté de manière identique les effets de la décision publique.

Le cas de Maisse, comme beaucoup d'autres en France (voir par exemple TORRE et al., 2006), constitue un bon exemple de conflits surgissant principalement après la déclaration d'utilité publique et pouvant durer de nombreuses années, y compris parfois après la réalisation du projet. Ces conflits mettent généralement en question la fiabilité de l'étude quantitative, et/ou la régularité formelle du déroulement de l'enquête publique. Ils révèlent que les oppositions aux projets d'infrastructures découlent à la fois des caractéristiques de la procédure juridico-administrative et de ses détours, ainsi que de l'évaluation quantitative des couts et des dommages éventuels. 
La route départementale $\mathrm{N}^{\circ} \mathrm{D} 837$ assure pour le Sud du département de l'Essonne l'important rôle de liaison entre Fontainebleau et l'autoroute A6 à l'Est et la RN 20 à l'Ouest. Elle relie également Ablis avec les autoroutes A10 et A11 via la RN 191. Traversant le cour de la commune de Maisse dans des conditions jugées incompatibles avec l'important écoulement du trafic routier - étroitesse de la voie, stationnement latéral autorisé et faible largeur des trottoirs dans le centre - elle devient dangereuse pour les piétons et génère de fortes nuisances de bruit et de pollution pour les riverains. Devant cet état de fait, le département de l'Essonne a projeté de réaliser une déviation de la RD837 contournant l'agglomération de Maisse.

Le projet de contournement, approuvé par le Conseil Général en 1999 et déclaré d'utilité publique par le Préfet en avril 2004, présente ainsi un intérêt incontestable pour la commune, mais également pour le département de l'Essonne. Il correspond pourtant à un conflit long d'une vingtaine d'années. En effet, si le désengorgement du centre ville s'avère une nécessité pour les habitants de Maisse et leurs voisins, la question de son tracé se révèle rapidement sensible et complexe.

En 1990, le Préfet lance un projet pour détourner la RD837 vers le Sud. Ce tracé suit la frontière de Maisse avec la commune voisine de Gironville-sur-Essonne et traverse une zone relativement peu peuplée. Validé par une déclaration d'utilité publique le 16 mai 1990, avant d'être prorogé par un arrêté préfectoral du 12 mai 1995 à la demande du Conseil Général, ce projet est enfin officiellement abandonné le 23 septembre 1999 par une délibération du département pour des raisons socioéconomiques, financières et environnementales. La même année, Maisse entre dans le périmètre du Parc Naturel Régional du Gâtinais français ${ }^{6}$ et la protection des espaces naturels conduit à l'abandon du tracé Sud. Un nouveau projet est envisagé, cette fois au Nord, où se sont développés entre temps de nouveaux lotissements résidentiels, habités par des riverains prévoyant que la déviation passerait au Sud.

Le conflit s'étend alors autour du choix du tracé Nord. D'un côté, les protagonistes de la déviation, à savoir la commune de Maisse, le département de l'Essonne et le Préfet, voudraient réaliser le plus tôt possible la déviation au nom de l'intérêt général. Le maire de Maisse a même interdit le passage des poids lourds au centre ville pour assurer la sécurité de ses habitants. De l'autre, les riverains s'opposent avec véhémence à la construction d'une route passant devant leurs propriétés nouvellement construites. L'Association contre la Déviation Du Nord de Maisse (ADDNM) est créée dans ce contexte. Née en 2003 au cours de l'enquête publique, elle regroupe des riverains s'opposant au tracé vers le Nord dans quatre communes : Courdimanche sur Essonne, Boutigny sur Essonne, Maisse et Mily la Forêt. L'ADDNM, dont l'objet est l'annulation du projet de déviation vers le Nord, est l'opposant principal tout au long de ce conflit.

Pour pouvoir comprendre l'histoire de ce conflit, notamment les raisons du choix du tracé Nord, il est nécessaire d'examiner les variantes présentées dans le dossier d'étude d'impact. La Figure 3 ci-dessous présente les quatre variantes A, B, C, et D proposées, qui se distinguent principalement par leurs distances au centre ville de 
Maisse. La variante D - la plus courte - passe au Sud de Courty et de la zone de forte densité de population de la commune. Deux variantes médianes - B et C - traversent Maisse par la zone résidentielle dans le Nord. Quant à la variante A - la plus longue elle contourne Maisse par sa frontière avec les communes de Courdimanche-surEssonne et de Boutigny-sur-Essonne, et traverse la zone nouvellement urbanisée mentionnée ci-dessus. Longue de 6,5 km, c'est la plus éloignée des habitations.

Finalement, c'est le tracé A, le plus lointain, qui est retenu par l'étude, au motif qu'il a le plus faible impact (acoustique et visuel) sur le cadre de vie des riverains. Il est également évident qu'il s'agit de la solution la moins coûteuse au regard de la difficulté d'expropriation.

\section{Figure 3 - Les variantes du tracé de la déviation selon le dossier d'enquête publique}

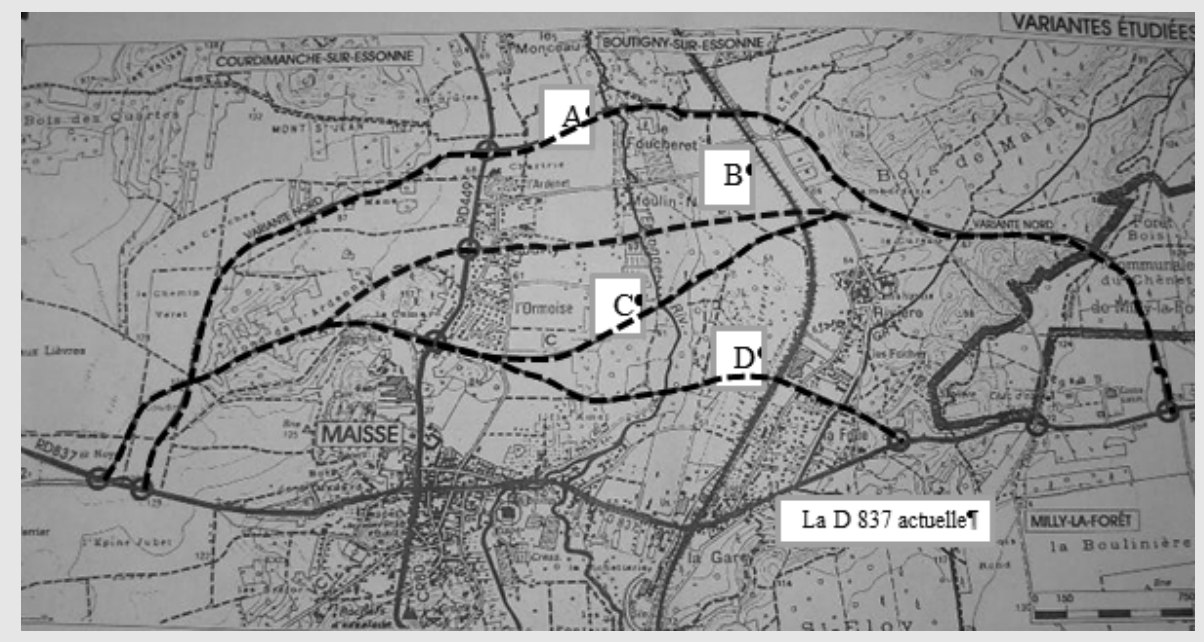

Observons le conflit par une analyse du contentieux ${ }^{7}$. La bataille juridique que mène l'ADDNM à l'encontre du projet, commencée en 2004, est toujours en cours. Sur ce plan, l'ADDNM a été rejointe par la commune de Courdimanche-sur-Essonne - également opposée à la déviation sur son territoire. Deux requêtes, respectivement de l'ADDNM et de la commune de Courdimanche, ont été déposées devant le Tribunal Administratif de Versailles, qui a statué en leur faveur. Mais le combat ne s'est pas arrêté là, puisque le Conseil Général de l'Essonne, le Préfet du département et le Ministre de l'Intérieur et de l'Aménagement du territoire ont fait appel par un recours devant la Cour administrative d'appel de Versailles Cette dernière a annulé le jugement du Tribunal administratif, ce qui a pour effet de maintenir le projet. Le conflit continue alors devant le Conseil d'Etat après l'appel de l'ADDNM. ${ }^{8}$ 
Afin de comprendre les causes du conflit, ainsi que les positions et les revendications des parties en présence, il est important d'entrer dans les arguments présentés. Nous ne retenons pas les motifs de «légalité externe» (incompétence de l'acteur du litige, vices procéduraux), pour nous concentrer sur les éléments qui portent sur le fond de l'affaire et éclairent le lien entre conflit et décision publique. Les principaux éléments de contestation sont de trois ordres :

- les fondements de l'utilité publique : les opposants au projet remettent en cause l'utilité publique, alors que les défenseurs du projet la défendent ;

- le calcul économique public: les opposants soutiennent que ce calcul est erroné ;

- l'optimalité de la décision publique. Cette question n'est pas été évoquée en tant que telle, mais fait partie des arguments de l'ADDNM, qui soutient que la décision en question n'est pas la meilleure.

Sur le premier point, l'argument central des riverains est que différents éléments de nature à modifier le caractère d'utilité publique du projet, en particulier sur le plan environnemental et paysager, n'ont pas été pris en compte dans l'arrêté préfectoral l'approuvant. Cet argument a été favorablement accueilli par le Tribunal Administratif: "l'impact paysager et écologique du projet apparaît important sur le site classé de la moyenne vallée de l'Essonne, protégé en raison de sa valeur paysagère et patrimoniale; deux zones naturelles d'intérêt écologique, faunistique et floristique (ZNIEFF) en seraient affectées; une chênaie pinède oligotrophe et un bois de chêne pubescent, qualifiés l'un et l'autre d'intérêt écologique exceptionnel par l'étude d'impact, seraient partiellement détruits; en outre, selon l'étude d'impact, le projet, surplombant les habitations de la vallée, impliqueraient des désagréments visuels pour les riverains. Au total, les atteintes à l'environnement et au paysage impliqués par l'opération paraissent excessifs au regard de l'intérêt de la déviation" (jugement 0404663, 2006).

Mais la Cour administrative d'appel n'a pas partagé ce point de vue. Elle considère que « la topographie des lieux marquée par une longue descente dans une rue étroite et animée de village avec un croisement régulé par un feu rouge en milieu de descente et en cœur d'agglomération induit un risque élevé d'accidents graves... que l'intérêt général attaché à l'objectif de réduction des nuisances pour l'ensemble des habitants de la commune...n'est pas contesté ». Cette discussion nous conduit au cœur du problème de l'appréciation qualitative de l'utilité publique, qui peut être formulée en termes d'arbitrage entre un enjeu environnemental et un enjeu de sécurité routière. D'un point de vue économique, cet arbitrage peut être exprimé en terme de monétarisation de la vie humaine et de la pollution; cependant, le juge administratif ne s'appuie pas sur ce point de vue et formule son diagnostic sur une appréciation qualitative, largement discrétionnaire, des enjeux en présence (sur ce point, cf. KIRAT (dir) 2005).

Sur le deuxième point, l'ADDNM soutient que les calculs économiques ont été basés sur des considérations contestables. En particulier, le coût de réalisation du 
projet serait sous-évalué de $40 \%$ du fait de l'omission de l'inflation dans le taux d'actualisation et de l'oubli d'un carrefour giratoire dans les ouvrages de construction. Le débat porte cette fois directement sur des éléments quantitatifs. Pour le Tribunal Administratif, ces points sont susceptibles de modifier le caractère d'utilité publique du projet, car selon la jurisprudence administrative : « une opération ne peut être déclaré d'utilité publique que si les atteintes à la proprprivée, le coût financier et éventuellement les inconvénients d'ordre social et les atteintes à d'autres intérêts publics qu'elle comporte ne sont pas excessifs eu égard à l'intérêt qu'elle présente ". Comme c'est systématiquement le cas dans les dossiers de DUP, le Tribunal Administratif a appliqué la théorie jurisprudentielle dite «du bilan», afin de montrer que le bilan du projet sera limité si l'on tient compte des «erreurs» de calcul économique ${ }^{9}$.

Même si cette "théorie du bilan " (Conseil d'Etat, Ville Nouvelle Est, 28 mai 1971) peut laisser penser que le juge administratif pèse les intérêts en présence et procède à une évaluation propre des coûts et avantages du projet sur lequel porte une déclaration d'utilité publique, on peut considérer que l'action de ce dernier reste circonscrite à la légalité " interne » et " externe ${ }^{10}$ de la décision contestée. En effet, selon cette jurisprudence administrative, une opération ne peut légalement être déclarée d'utilité publique que si les atteintes à la propriété privée, le coût financier et éventuellement les inconvénients d'ordre social et les atteintes à d'autres intérêts publics qu'elle comporte ne sont pas excessifs eu égard à l'intérêt qu'elle présente. Cet arrêt fut innovant dans la mesure où il élargit la sphère d'appréciation de l'utilité publique : désormais, le juge s'attache à déterminer si le coût financier de l'opération et les inconvénients qu'elle implique ne sont pas disproportionnés relativement à ses avantages. Cependant, l'application de la théorie du bilan n'est pas sans limites, d'abord parce que le juge n'a pas compétence pour statuer sur l'opportunité du choix par l'administration d'une localisation ou d'un tracé parmi un ensemble d'alternatives, ensuite parce que la " grille d'analyse » de l'utilité publique fondée sur une mise en balance des avantages et des coûts et inconvénients est peu élaborée et, selon le Conseil d'Etat, a débouché sur un nombre relativement limité d'annulations. ${ }^{11}$

La Cour administrative d'appel retient cependant une autre manière de juger ce bilan. Sans entrer dans les erreurs de calcul telles qu'elles sont présentées, elle soutient que " ni le coût élevé, ni les inconvénients pour l'environnement et l'agriculture que présente l'opération ...ne sont d'une importance telle qu'il aient pour effet de lui (le projet) retirer le caractère d'utilité publique ». Cette réponse déplace ainsi le débat sur les fondements de l'utilité publique : on passe d'une utilité fondée sur le calcul quantitatif à une utilité fondée sur des appréciations qualitatives, qu'il s'agisse de la sécurité routière ou du besoin d'aménagement exprimé par les pouvoirs publics. Cela revient à faire prévaloir la sécurité et le trafic routier au-delà du calcul des coûts en termes monétaires. La balance entre des éléments qualitatifs et quantitatifs selon les deux juridictions administratives met ainsi en évidence toute la difficulté de juger de la valeur d'un projet en termes d'utilité publique.

Concernant le troisième point, il faut noter que l'ADDNM ne s'oppose pas à la déviation, mais soutient que celle-ci devrait passer au Sud et non pas au Nord. 
Plus long en distance, le tracé Nord s'annonce non seulement plus coûteux, mais aura également plus d'impact sur le cadre de vie résidentiel que l'itinéraire Sud. L'association évoque ainsi une comparaison des tracés Sud et Nord sur les mêmes critères de choix. Or, cette demande est rejetée par la Cour administrative d'appel, car « au regard au délai écoulé entre l'abandon du projet de contournement par le sud....le premier projet (par le sud) ne pouvait être regardé comme un "parti envisagé » au sens des dispositions précitées de l'article R.11-3 [nda : du Code de l'expropriation pour cause d'utilité publique]».

Cette position de la cour administrative d'appel rappelle que les tribunaux ne sont pas des lieux de justification d'une décision publique au regard des critères politico-économiques, dans la mesure où la vocation du juge se limite à " répondre juridiquement à des demandes exposants des litiges (...) ou à des requêtes résultant de la lésion d'intérêts » (KIRAT et MELOT, 2005). De plus, ils ne sont pas juges de l'opportunité des choix publics et, de surcroît, le fait que les requêtes soient forcément basées sur des décisions administratives par nature ponctuelles a pour effet de ne jamais donner à voir l'ensemble du processus au juge administratif.

A ce stade, nous constatons que le processus décisionnel ne laisse pas de place pour douter de l'optimalité d'une décision : le calcul économique mené en amont du projet statue sur son intérêt public, et la procédure d'enquête publique a vocation d'en donner confirmation. Il en découle une double limite : d'abord, même si la procédure de consultation du public est organisée formellement, elle ne garantit pas que tous les intérêts s'expriment ; ensuite, le processus ne permet pas l'élaboration ou la discussion d'alternatives. En effet, le commissaire enquêteur n'a pas vocation à tracer toutes les possibilités de réalisation d'un projet, mais de statuer sur un projet concret donné.

Les éléments qui précèdent éclaircissent les origines et les raisons d'un conflit mené pendant de nombreuses années contre les Pouvoirs publics. L'analyse du contentieux montre que la possibilité de vérifier avec impartialité l'optimalité de la décision publique n'existe pas. Puisque l'enquête publique ne permet pas d'atteindre une acceptabilité sociale qui ne soit pas entachée d'un sentiment d'injustice (RAux et al. 2004 ; RAux et Souche 2006), elle provoque oppositions et conflits aux décisions d'implantations d'infrastructures. On se trouve sans doute ici aux limites de la théorie économique, tant ces oppositions peuvent se produire dans une situation jugée optimale (fut-elle de second rang) par le calcul public ${ }^{12}$ et ressortir alors de critères qui ne sont plus totalement justiciables de ceux retenus traditionnellement par les pouvoirs publics en matière de décision de construction ou de modifications d'infrastructures. 


\section{- 5 - \\ Conclusion}

L'objectif de cet article était de présenter et d'analyser les conflits d'infrastructures en Ile-de-France et, au-delà, de jeter un éclairage sur le processus de la décision publique en la matière, ainsi que sur le rôle joué par les conflits dans ce processus. L'étude appliquée, menée sur les données du contentieux, nous a permis de mettre en évidence un certain nombre d'élément saillants. Les conflits d'infrastructures présentent les caractéristiques suivantes en Région Ile de France :

- ils sont essentiellement localisés dans les espaces périurbains et dans des communes à la population relativement aisée, distantes d'une trentaine de kilomètres du cœur de l'agglomération parisienne,

- ils sont l'expression d'une prise de parole de groupes de riverains, désireux de faire entendre leur voix et leur opposition face à des projets qui ne leur paraissent pas acceptables en l'état,

- ils sont l'écho de la lutte vive entre besoin d'urbanisation et besoin de préservation de l'espace qui se joue en région Ile-de-France, - ils révèlent que tous les territoires n'ont pas la même capacité de réaction à l'installation des infrastructures en raison des différences socioéconomiques des populations qui les occupent,

- ils sont révélateurs d'oppositions concernant des projets considérés comme utiles à l'intérêt général par leurs porteurs (généralement les décideurs publics) et faisant l'objet de contestations locales par des riverains soucieux de la protection et des atteintes à leur cadre de vie,

Par ailleurs, ces conflits mettent en évidence la complexité, mais aussi les ambiguïtés du processus de décision publique, qui se trouve confrontée à un double dilemme : l'arbitrage entre les dimensions qualitatives et quantitatives des projets d'une part, et la difficile soudure entre le travail réalisé au sein de l'administration et la manière dont les projets sont reçus par les populations concernées ou riveraines. Tant et si bien que les conflits finissent par entrer dans le jeu de la décision, par la mise en place d'un processus d'essais et d'erreurs des propositions de construction et de modification d'infrastructures. Vient s'ajouter à cet équilibre complexe la question des échelles spatiales. Quelle est l'échelle pertinente en matière de décision publique entre les exigences d'espace d'une Région Capitale et les attentes de territoires de taille plus restreintes, mais dans lesquels vivent des populations fermement décidées à défendre leur environnement et leur cadre de vie? 


\section{Bibliographie}

AGRESTE (2011) L'utilisation du territoire. Les paysages agricoles dominent toujours le territoire français. AGRESTE Primeur 60 (avril) :1-4.

BLATRIX C (2009) Cadrages, recadrages et hors-champ du débat public : le cas de l'assainissement en Ile-de-France. In : Ville éphémère, ville durable. Nouveaux usages, nouveaux pouvoirs. L'œil d'or, Paris.

BLATRIX C (1997) Faut-il un public à l'enquête publique ? Les paradoxes d'une procédure de démocratie participative. Techniques, Territoires et Sociétés 34 : 101-110.

Blatrix C, Blondiaux L, Fourniau J-M, Heriad-Bubreuil B, Lefebvre R, Revel M (2007) Le débat public: une expérience française de démocratie participative. La Découverte, Paris.

BOITEUX M (2001) Transport : Choix des investissements et coûts des nuisances. Rapport du Commissariat général du plan, Paris.

Bouba-Olga O, Boutry O, Rivaud A (2009) Un approfondissement du modèle Exit-Voice par l'Economie de la Proximité. Nature, Sciences et Sociétés 17 (4) : 381-390.

Bourdin A, Lefeuvre M-P, Mele P (dirs.) (2006) Les règles du jeu urbain, entre droit et confiance. Descartes et Cie, Paris.

CADORET A (2006) Conflits d'usage lié à l'environnement et réseaux sociaux: enjeu d'une gestion intégrée. Le cas du littoral du Languedoc-Roussillon. Thèse de Doctorat en géographie, Université de Montpellier III.

CARON A, TORRE A (2006) Quand la proximité devient source de tensions : conflits d'usages et de voisinage dans l'espace rural. Développement Durable et Territoires 7, revue en ligne.

CENTRE D'ANALYSE STRATÉGIQUE (2012) Les nouvelles mobilités dans, les territoires périurbains et ruraux. Collections \& Rapports, Rapport de la mission dirigée par Olivier Paul-Dubois-Taine, CAS, Paris.

CHARLIER B (1999) La défense de l'environnement: entre espace et territoire. Géographie des conflits environnementaux déclenchés en France depuis 1974. Thèse pour le doctorat en géographie, Université de Pau et des pays de l'Adour.

DARLY S (2009) Faire coexister ville et agriculture au sein des territoires périurbains. Antagonismes localisés et dynamiques régionales de la conflictualité. Thèse de Doctorat de l'Ecole de Hautes Études en Sciences Sociales, Paris.

DARLY S, TORRE A (2008) Conflits liés aux espaces agricoles et périmètres de gouvernance en lle-de-France (résultats à partir d'analyses de la presse quotidienne régionale et d'enquêtes de terrain). Geocarrefour 83 (4) : 307-319.

DOWDiND K, JOHN P, Mergoupis T, VUGHT M.V (2000) Exit, Voice, Loyalty: analytic and empirical developments. European Journal of Political Research 37 : 469-495.

Doron M (2007) Conflits et réseaux d'acteurs dans la construction d'espaces périurbains : analyse du jeu des acteurs dans deux processus de développement dans les régions de Montréal et de Montpellier. Communication aux colloques de la Relève, 4e édition: Des acteurs qui façonnent le territoire. Université de Montréal, 17 et 18 Mai.

FABUREL G (2003) Lorsque les territoires locaux entrent dans I'arène publique. Comparaison internationale des conflits liés au bruit des avions. Espaces et Sociétés 155 : 123-146.

GIBLIN J-P (2004) Faut-il un élargissement des modes d'évaluation des investissements publics ? Communication aux Journées de l'AFSE Economie et aide à la décision publique, 18-19 mai.

Gilly F (2007) Paris, une Métropole dans le monde, mise en perspective des savoirs. Etude exploratoire pour la DIACT, Université de Lille, Lille.

Guesnerie R (2006) De l'utilité du calcul économique public. Économie \& Prévision 175-176 : 1-15.

HIRSCHMAN A O (1970) Exit, Voice and Loyalty. Responses to decline in firms, organizations and states. Harvard University Press, Cambridge (MA).

Hostiou J-C, HeLIN R (1993) Droit des enquêtes publiques. Ed. Le Moniteur, Paris.

IAURIF (2001) Mode d'occupation du sol. Note rapide de I'IAURIF (282). 
JEANNEAUX P (2006) Les conflits d'usage dans les zones périurbaines et rurales françaises - Une approche par l'analyse économique de la décision publique. Thèse de doctorat en Sciences Economiques, Université de Bourgogne, Dijon.

JeANNEAux P, PERIER-CORNet P (2009) La contestation locale des décisions publiques et les conflits d'usage dans les espaces ruraux et périurbains français. Economie Appliquée 62 (4) : 157-182.

Joerin F, Pelletier M, Trudelle C, Villeneuve P (2005) Analyse spatiale des conflits urbains. Enjeux et contextes dans la région du Québec. Cahiers de Géographie du Québec 49 (138) : 319-342

KIRAT T, TORRE A (dirs.) (2006) Conflits d'usage et dynamiques spatiales : les antagonismes dans l'occupation des espaces ruraux et périurbains (I). Géographie, Economie, Société 8.

KIRAT T, MELOT R (2006) Du réalisme dans I'analyse économique des conflits d'usage : les enseignements de I'étude du contentieux dans trois départements français (Isère, Loire-Atlantique, Seine-Maritime). Développement Durable et Territoires 7 (http://developpementdurable.revues.org/document2574.html)

LECOURT A (2003) Les conflits d'aménagement - Analyse théorique et pratique à partir du cas de Breton. Thèse de Doctorat en Géographie, Université de Rennes II.

KIRAT T (dir.) (2005) Economie et droit du contrat administratif. La Documentation française, coll. Perspectives sur la justice, Paris.

KIRAT T, LEVRATTO N (2008) Tous égaux face aux nuisances des infrastructures de transport ? Le calcul économique et le droit administratif au défi de l'équité. Géographie, Economie, Société 10 : 350-364.

MATHIEU N H (2005) La population francilienne au janvier 2005. Document de l'INSEE - Ile de FranceFaits et Chiffres 112 1-4.

MAurice J, CROzet Y (2007) Le calcul économique dans le processus de choix collectif des investissements de transport. Economica, Paris.

Mele P, Larrue C, RoseNberg M (2004) Conflits et Territoires. PUFR, Tours.

MELOT R (2009) De la gestion des espaces au projet de territoire : les enjeux politiques d'un changement de paradigme juridique. L'année sociologique 59 (1) : 177-199.

Meriaux A, KaSt R, LAPIED A (2002) Prévoir l'impact d'un projet d'équipement. Economie publique 1 (10) : 39-49.

O'Lear S, Diehl P.F, Frazier D V, AlleE T L (2005) Dimensions of territorial conflict and resolution: tangible and intangible values of territory. GeoJournal 64: 259-261.

ORIZET F R (2003) Mobilité. Réflexions sur l'avenir de l'Ile-de-France, Direction Régionale de l'Equipement, Paris.

PHAM H V, KIRAT T (2008) Les conflits d'usage des espaces périurbains et le contentieux administratif. Le cas de la région Ile-de-France. Revue d'Economie Régionale et Urbaine 5 : 671-700.

PhAm H V, TORRE A (2012) La décision publique a l'épreuve des conflits, un cadre d'analyse des processus professionnels au regard de l'expression de l'opposition. Revue d'Economie Industrielle 137.

Raux C, SOuChe S, VASKOVa L (2007) Les figures de l'acceptabilité. In : MAUriCe J, Crozet Y. (dirs.) Le calcul économique public. Economica, Paris.

SEGESA/DREIF/DRIAF (2005) Dynamique territoriale de l'agriculture et de l'espace rural en Ile-deFrance. SEGESA, DREIF et DRIAF, Paris [http://driaf.ile-de-france.agriculture.gouv.fr/IMG/pdf_Partie1_Dynamiq_territor_agr_ildF_cle884a36.pdf].

Torre A, aznar O, Bonin M., Caron A, Chia E, Galman M, Lefranc C, Melot R., Guerin M, Jeanneaux P, Kirat T, PaOli J -C, Salazar M -I, Thinon P (2006) Conflits et tensions autour des usages de I'espace dans les territoires ruraux et périurbains. Le cas de six zones géographiques françaises. Revue d'Economie Régionale et Urbaine 3 : 411-450.

Torre A, Melot R, Bossuet l, Cadoret A, Caron A, Darly S, Jeanneaux P, Kirat T, Pham H V (2010) Comment évaluer et mesurer la conflictualité liée aux usages de l'espace ? Eléments de méthode et de repérage. VertigO 10 (1). Mis en ligne le 30 avril 2010. http://vertigo.revues.org/9590.

TRUDELLE C (2003) Au-delà des mouvements sociaux : une typologie relationnelle des conflits urbains. Cahiers de Géographie du Québec 47 (11) : 223-242. 
WESTER-HERBER M (2004) Underlying concerns in land-use conflicts - the role of place-identity in risk perception. Environmental Science \& Policy 7 : 109-116.

YOUNG D R (1976) Consolidation or diversity: choices in the structure of urban governance. American Economic Review 66 (2) : 378-385.

\section{Notes}

1 - Not in my backyard.

2 - Nous remercions vivement Marina GALMAN, qui a été la cheville ouvrière de ce projet.

3 - Sur ce point, voir KirAT et LeVRATTO (2008 : 360-361)

4 - Le terme "événement" ne recouvre pas exclusivement des actes techniques avérés, tels que la construction d'un immeuble ou d'une bretelle d'autoroute, un remembrement, la pollution d'une rivière... ; il peut également s'agir d'une décision censée avoir des effets dans le futur.

5 - Le terme "événement" ne recouvre pas exclusivement des actes techniques avérés, tels que la construction d'un immeuble ou d'une bretelle d'autoroute, un remembrement, la pollution d'une rivière... ; il peut également s'agir d'une décision censée avoir des effets dans le futur.

6 - Le PNR du Gâtinais français couvre 57 communes, dont Maisse et ses communes voisines.

7 - Dans le cadre de l'étude, nous avons également réalisé des entretiens et un recensement de la presse. Ces sources complémentaires confirment les résultats de l'analyse du contentieux ici présentée.

8 - A la date où nous écrivons, le Conseil d'Etat n'a pas rendu son jugement.

9 - Selon la jurisprudence administrative, dite "théorie du bilan", une opération ne peut légalement être déclarée d'utilité publique que si les atteintes à la propriété privée, le coût financier et éventuellement les inconvénients d'ordre social et les atteintes à d'autres intérêts publics qu'elle comporte ne sont pas excessifs eu égard à l'intérêt qu'elle présente. Selon l'appréciation sommaire des dépenses, le coût financier au regard du trafic attendu dépasse déjà l'intérêt de l'opération, ce qui est de nature à lui retirer son caractère d'utilité publique.

10 - La légalité interne concerne la question de savoir s'il y a eu violation de la loi, sous la forme d'erreurs de droit, de qualification juridique ou de fait ; la légalité externe répond à la question de savoir si la décision prise l'a été de manière régulière, indépendamment de son contenu.

11 - http://www.conseil-etat.fr/cde/node.php?articleid=1307

12 - L'inverse est vrai, l'acceptabilité pouvant correspondre à une situation jugée non optimale par le calcul public, en cas d'absence de démocratie par exemple... 